\title{
Die zweite Natur
}

Liebe Leserinnen, liebe Leser,

der Computer - programmierte Schaltkreislogik - ist mit nichts vergleichbar. In vieler Hinsicht - Leistung, Flexibilität, Genügsamkeit - übertrifft er das Gehirn, obwohl er, wie die Technik überhaupt, etwas Hirngeborenes ist. Anders als die herkömmliche Technik entlastet er aber nicht die Physis sondern das Gehirn, seinen Schöpfer. So hilft er ihm auch dabei, ihn bzw. sich selbst zu vervollkommnen. Mit dieser Rückkopplung ergibt sich für den Computer ein exponentielles Wachstum. Er wird zudem auch vielseitiger, variationsreicher, anpassungsfähiger und expandiert ins Globale. Er verleiht Gegenständen Individualität und praktische Intelligenz und macht sie fähig, untereinander und auch mit dem Menschen zu kommunizieren. Dem Menschen entsteht damit eine neue Welt, eine Art selbst geschaffene zweite Natur. Er nimmt sie in Anspruch. Sie macht ihm das Leben leichter und angenehmer, gibt ihm Lebensqualität. Aber damit nimmt sie ihn auch in Abhängigkeit.

Diese zweite Natur entwickelt sich mit dem Fortschritt des Computers. Dabei beeinflusst, wie oben gesagt, das bereits Geleistete das Hinzukommende. So verfestigt sich der Fortschritt zunehmends und die zweite Natur gewinnt eigengesetzlich Konturen. Sie wird dem Menschen vertraut. Ihre per Computer geleistete Zuwendung erscheint ihm als so natürlich wie eine Handreichung durch einen Freund.

Von all dem ist der Mensch doppelt betroffen: Auf der aktiven Seite entwickelt, betreibt und kontrolliert er den Computer und seine Dienste. Auf der passiven benötigt und konsumiert er sie. Als Individuum kann er im Prinzip beide Rollen einnehmen. In der Regel spezialisiert er sich aber und ist entweder Anbieter oder Konsument. Daraus ergibt sich eine Struktur der Machtverteilung. Als Anbieter hat er Macht über den Konsumenten. Wie die Wirtschaftsentwicklung zeigt, entstehen auf der aktiven Seite Unternehmen, die ihre Dienste auf den Markt bringen, um einen Bedarf zu decken. Mit dem Bedarf geraten die Konsumenten in eine Abhängigkeit, die zudem von computerunterstützter Werbung verstärkt wird. Sie werden in die passive Rolle des kleinen Mannes gedrängt, der bei Laune gehalten werden kann. Diese Polarisierung der Machtverhältnisse ist zum guten Teil auf die oben erwähnte Rückkopplung zurückzuführen. Die Macht wächst mit dem Computer und mit der zweiten Natur.

Nun Spekulatives: Die zweite Natur wird dem Menschen vertraut. Er gewinnt ein intimeres Verhältnis zu den Dingen, mit denen er kommuniziert und fühlt sich selbst als Bestandteil der Zweiten Natur. Er ordnet sich ein und empfindet keinen operativen Unterschied zwischen Menschen und Dingen. Er hält auch seine menschlichen Kommunikationspartner wie die dinglichen für programmierbar. So vermittelt ihm die zweite Natur ein besonderes Gemeinschaftsgefühl. Über sie kann er im Sinne der oben aufgezeigten Macht beeinflusst werden.

Das ist zwar Spekulation, aber man muss in Betracht ziehen, dass Gemeinschaftsgefühl und Lebensgefühl mit der Zeit gehen. Die Probleme werden anders und werden von der Gesellschaft auch anders bewertet. Vor allem in Umbruchzeiten wie der gegenwärtigen können die Veränderungen durchgreifend sein und zu schwer begreifbaren altersbedingten Unterschieden im Lebensgefühl führen. Selbst zwischen den heute gemeinsam lebenden Generationen gibt es diesbezüglich deutlich erkennbare Unterschiede, immerhin erkennbare. Ein Greis von heute kann bis zu sechs Generationen überblicken und Unterschiede im Lebensgefühl der Generationen wahrgenommen haben. Darüber hinaus sind ihm die weiteren entrückt. Niemand kann heute voraussehen, wie sich eine so entrückte Generation mit dem Leben arrangieren, wie sie sich speziell auf die zweite Natur einstellen wird.

Den Anfang, den Veränderungen zu begegnen, liebe Leserinnen und Leser, hat der Datenschutz gemacht. Er stellt sich an, das Sammelbecken für hinzukommenden Bedarf zu werden, aber er kann auch gegenüber den noch bevorstehenden Problemen verblassen.

Mit freundlichen Grüßen, Ihr

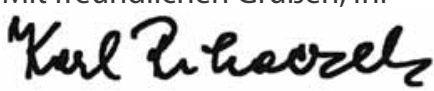

